\title{
Improving stability, robustness and performance of Laser systems
}

\author{
Andres Guesalaga ${ }^{1}$, Benoit Neichel ${ }^{2}$, Maxime Boccas ${ }^{2}$, Celine D'Orgeville $^{2}$, Francois Rigaut ${ }^{2}$, Dani \\ Guzman $^{1}$ and Jaime Anguita ${ }^{3}$ \\ ${ }^{1}$ Universidad Católica de Chile, 4860 Vicuna Mackenna, Casilla 7820436, Santiago, Chile \\ ${ }^{2}$ Gemini Observatory Southern Operations Center, Colina el Pino s/n, Casilla 603, La Serena, Chile \\ ${ }^{3}$ Universidad de Los Andes, San Carlos de Apoquindo 2200, Las Condes, Santiago, Chile \\ *corresponding author: aguesala@ing.puc.cl
}

\begin{abstract}
The vast majority of large telescopes are now equipped with Adaptive Optics (AO) systems, and many use lasers to create artificial stars (laser guide stars, LGS). Despite the significant advances in the use of LGS for AO, some problems persist during the operations. In particular, achieving a satisfactory performance in terms of on-sky laser power and beam quality usually requires frequent and complex alignments of the laser system, beam transfer optics and launch telescope. To provide easier calibrations and faster pre-setting of the LGS facility during routine operations, we propose the introduction of active elements (deformable mirrors) in the laser beam before it is propagated to the sky. The paper studies an AO configuration with two deformable mirrors to correct for quasi-static and dynamic aberrations. The problem of determining the correction phases to apply to the deformable mirrors is particularly challenging due to the highly nonlinear problem and the possible appearance of branch points. We propose an iterative method based on a phase retrieval algorithm that uses a weighted least squares unwrapper to avoid branch points. Simulations are performed aiming to a future implementation in the Gemini Multi-conjugate-adaptive-optics System (GeMS). Results show that the technique is accurate and robust, with a reasonable convergence speed.
\end{abstract}

Keywords: Adaptive optics, laser systems, deformable mirrors, phase retrieval

\section{INTRODUCTION}

In all major ground-based astronomical observatories, Adaptive Optics (AO) has become an intrinsic technique to bring scientific observations closer to the diffraction limit of the optical instruments. The technique requires guide stars with a specified minimum brightness to act as reference and to probe the atmospheric turbulence, providing the information to correct the scientific images by removing the aberrations induced by the atmosphere on the optical wavefront.

The minimum brightness of these reference stars typically varies between 12 and 16 magnitudes in the optical (depending on the telescope and position in the sky), imposing the hardest limitation on the use of AO in astronomical instruments due to the scarcity of Natural Guide Stars (NGS) occurring near the narrow field of views of the scientific targets.

Angular anisoplanatism also impacts the AO technique negatively, and correction with NGS can only be performed over a very small field of view (typically a fraction of an arcminute) around the scientific target. This effect further limits the number of targets that can be observed with this technique, that together with the brightness restriction, reduces the sky coverage to values that would make AO almost futile. For example, to compensate images at a wavelength of $2.2 \mu \mathrm{m}$ using a reference source with magnitude $\mathrm{m}=14$ and isoplanatic angle of 10 arcseconds, the sky coverage is approximately $0.1 \%{ }^{1}$.

An artificial guide star can be produced by either Rayleigh scattering in the lower layer of the atmosphere (below $20 \mathrm{~km}$ altitudes) or by excitation and subsequent spontaneous decay of the atoms present at the mesospheric sodium layer (at about $92 \mathrm{~km}$ altitude). The emission of interest occurs in the sodium $\mathrm{D}_{2 \mathrm{a}}$ line.

After a difficult introductory phase of this technology filled with technical and operational problems, the astronomical community has gained significant experience and today a new generation of these systems is being developed and tested to be used in more challenging observational strategies such as wide-field AO system. These systems will include multiple lasers for atmospheric turbulence tomography, multi-conjugate AO and multi-object AO, among others. Some

Adaptive Optics Systems III, edited by Brent L. Ellerbroek, Enrico Marchetti, Jean-Pierre Véran, Proc. of SPIE Vol. 8447 , $84474 \mathrm{M} \cdot$ ? 2012 SPIE $\cdot$ CCC code: $0277-786 / 12 / \$ 18 \cdot$ doi: $10.1117 / 12.924908$ 
examples of these initiatives are the MMT's laser adaptive optics system ${ }^{2}$, the Gemini's $\mathrm{GeMs}^{3}$, the VLT's adaptive optics facility ${ }^{4}$ and the Keck $\mathrm{NGAO}^{5}$. Furthermore, in the next decade the world will see the future generation of telescopes with an even stronger demand on laser capabilities. The $\mathrm{LBT}^{6}, \mathrm{TMT}^{7}, \mathrm{E}^{-\mathrm{ELT}^{8}}$, and $\mathrm{GMT}^{9}$ giant observatories will be equipped with these facilities in order to generate the required artificial asterisms for the wide-field AO observations.

Despite the significant advances in their use, some problems persist during operation and in the majority of the aforementioned telescopes, the laser systems have been available less than $50 \%$ of the time ${ }^{10}$. Achieving a satisfactory performance in terms of on-sky laser power and beam quality usually requires frequent and complex alignment of the laser system, beam transfer optics and launch telescope.

During operations, overheads to tune the laser guide star facility can take a substantial part of the total observation time $^{10}$. Moreover, the final AO performance is directly (and dramatically) affected by a drift of the laser beam quality, which is usually sensitive to temperature and environmental conditions.

As in other areas where these devices are used, such as weapons systems and recently in free space communications, the goal is always to concentrate as much power as possible in the smallest possible spot of the illuminated target, the latter being either a space vehicle, a communication link receiver or the atmosphere $e^{11-14}$.

There are two main sources of wavefront aberrations occurring on the laser beam that could degrade the spot quality: (i) calibration miss-adjustments of the laser source, beam transfer optics and launch system and (ii) aberrations induced by the atmosphere along the wavefront optical path.

In the first case, the errors in both amplitude and phase can be corrected by some form of wave front probing or beam intensity profile measurement and they can be understood as static phenomena. Correcting the second type of aberrations is far more complex, because it is highly dynamic and includes exogenous factors difficult to measure and compensate. If the turbulence is located outside the near field of the laser launch aperture, both the phase and the amplitude of the wave will be corrupted at the target point. Correcting for these two effects of atmospheric turbulence has generated a significant amount of interest during the last two decades in the fields of free space communications and weapon systems ${ }^{11-14}$. However, no major effort is known regarding laser beam correction in beacon generation for astronomical observations. This paper aims to enhance the quality of the laser beams in the far field by developing new methods in the near field and conjugate fields of laser guide star facilities.

Our main goal is to reduce the spot size of the laser guide stars in order to increase the performance of the current and future laser $\mathrm{AO}$ systems, delivering the best astronomical images.

A first possible configuration to improve the laser beam would entail correcting the amplitude or the phase alone. There is substantial work reported in the literature regarding amplitude modulation, and particularly in the industrial sector ${ }^{15-18}$. Although attractive to applications in the near field, this approach may induce a large scintillation and broadening of the spot at targets in the far-field, due to uncontrolled phase distortion at the pupil. This approach also wastes a significant amount of the laser energy.

When properly performed, phase modulation of a beam can remap the intensity in a way that is virtually lossless. Unfortunately, it would not provide full optical field conjugation and in many cases, correcting for amplitude is a must.

As an illustrative example, Fig. 1 shows an early 2011 amplitude profile of the Gemini South 50W laser beam in the near field (left) and the corresponding profile in the far field (right). The near-field and far-field profiles were measured at the output of the Gemini South laser system, prior to propagation through the beam transfer optics of the laser guide star facility ${ }^{3}$. The amplitude profile is clearly distorted and at the same time the far field laser spot is elongated. 

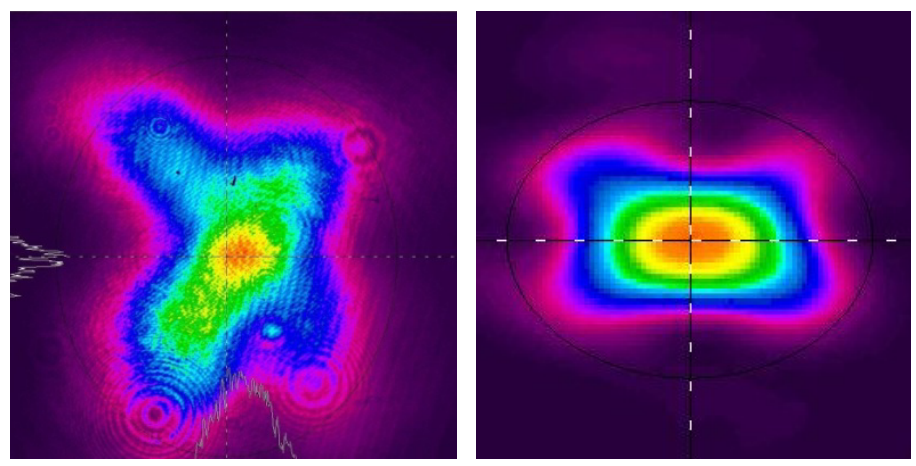

Figure 1. Near field (left) and far field (right) at the Gemini south laser facility

Correcting for both amplitude and phase aberrations is thus required, and is referred to as optical field conjugation ${ }^{13}$. The amplitude and phase of an optical wavefront can be controlled by liquid-crystal spatial light modulators ${ }^{19,20}$, but their low energy tolerance and efficiency make them an unacceptable option. It is highly desirable then, to achieve amplitude and phase control with reflective elements. Deformable mirrors (DM) are appealing candidates to fulfill these goals.

In this project we propose the use of multiple mirror configurations to correct the outgoing wavefront in a laser projection system.

\section{SUMMARY OF APPROACH}

One of the first approaches to tackle phase and amplitude correction simultaneously was proposed by Roggemann and $\mathrm{Lee}^{13}$. Figure 2 shows a block diagram for this correction system. The phase and amplitude corruption induced in the optical path from the target are measured with a suitable device, such as a Hartmann sensor. The wavefront sensor outputs are processed to compute the amplitude and the phase of the field in the telescope aperture. A phase-retrieval method processes the amplitude and phase measurements to determine the phase deformation to be applied to DM2 and DM1. The computer calculates the distortion to apply to the DM2 via the phase-retrieval algorithm, which should be a close match to the desired amplitude profile when the light hits the DM1. In this configuration, the DM1 lies in the Fraunhoffer diffraction region of the DM2, thanks to the Fourier-transforming mirror shown in Fig. 2 and the DM1 is assumed to be optically conjugated to the pupil of the beam projection system. The goal of the DM1 is to correct the phase distribution of the output beam so that the field is conjugated to the incident field from the beacon as it leaves the aperture.

This kind of dual-DM systems has been used during the last two decades in the military and free space communication fields, but no significant efforts are known to the authors regarding optical field conjugation in the astronomical field.

First, turbulence effects in free space communication and defense are more important due to the stronger turbulence in the beam effective path that in many cases is longer and horizontal, corresponding to Rytov variance values well above unity $^{21}$. Second, the beacon in these two fields is extended over wider areas than in the case of Rayleigh or sodium layer scattering, generating spots that differ from point-like beacons, making tilt and higher order error difficult to estimate. Third, in the case of sodium layer and Rayleigh scattering, the spot can have a thickness that ranges from some tens of meters to several kilometers, which differs from the receiver or target rough surface in the communication and weapon applications ${ }^{1}$.

Finally, the nonlinear interaction of the laser with the medium, usually referred to as blooming, may be considered negligible in laser guide stars (LGS), but it is a major source of concern in high power laser systems used in defense ${ }^{22}$.

Based on these factors, this article studies the optical configuration in Fig. 3 and related algorithms to improve the performance of the laser projection system for LGS using simulation tools. 


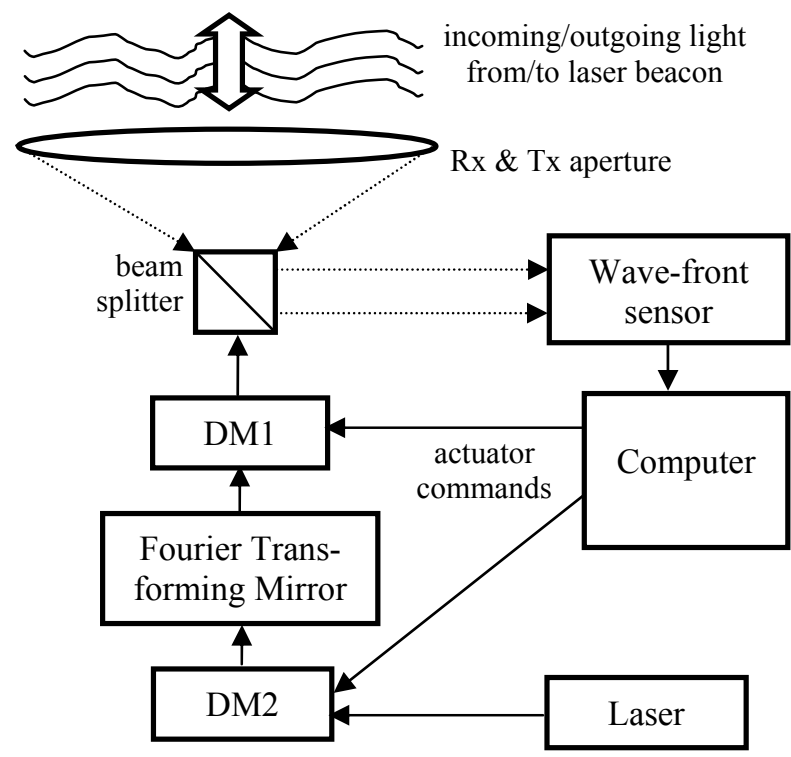

Figure 2. A classical monostatic laser projection configuration with two deformable mirrors ${ }^{13}$

\section{PHYSICS-BASED DEVELOPMENT OF A CONTROL ALGORITHM FOR THE TWO-DM SYSTEM}

A double deformable mirror correction is considered and the corrupted wavefronts from the beacon and laser source are assumed to be measured with wave-front sensors (WFS).

In Fig. 3 the wavefront incident upon the aperture from the beacon $\left(U_{b}\left(x_{f}\right)\right)$ is represented by:

$$
U_{b}\left(x_{f}\right)=A_{b} \exp \left[j \varphi_{b}\left(x_{f}\right)\right]
$$

where $x_{f}$ is a position vector in the telescope pupil, $A_{b}$ is the amplitude of the star (either natural or laser), assumed constant over the aperture and $\varphi_{b}\left(x_{f}\right)$ is the phase aberration of the incoming field due to turbulence. Let the wave-front of the transmitted field be represented by:

$$
U_{t}\left(x_{f}\right)=B\left(x_{f}\right) \exp \left[j \varphi_{t}\left(x_{f}\right)\right]
$$

where $B\left(x_{f}\right)$ and $\varphi_{t}\left(x_{f}\right)$ represents the amplitude and phase of the field reflected from DM1. In the laser beam projection context, optical field conjugation means that $B\left(x_{f}\right)$ and $\varphi_{t}\left(x_{f}\right)$ are adjusted so that ${ }^{23}$ :

$$
\varphi_{t}\left(x_{f}\right)=-\varphi_{b}\left(x_{f}\right)
$$

and

$$
B\left(x_{f}\right) \propto A_{b}
$$

In the general case, equation (4) seems unrealistic due to diffraction and laser power losses caused by the abrupt aperture stop, so $B\left(x_{f}\right)$ should be shaped to generate near and far field optical responses that avoid the latter problems or take into account the optics of the launcher system, e.g. Gaussian amplitude or annular shape amplitude for Cassegrain types of projection systems. This amplitude shaping is later discussed in the paper.

As mentioned before, one of the objectives of this work is to correct for laser amplitude and phase aberrations at the laser source. The laser beam hitting DM2 is given by,

$$
U_{L}\left(x_{f}\right)=A_{L}\left(x_{f}\right) \exp \left[j \varphi_{L}\left(x_{f}\right)\right]
$$

The amplitude of the field illuminating DM1 $\left(U_{l}\left(x_{f}\right)\right)$ will be given by the original laser field and the phase deformation applied to DM2: 


$$
U_{l}\left(x_{f}\right)=U_{L}\left(x_{f}\right) \exp \left[j \varphi_{2}\left(x_{f}\right)\right]
$$

where $\varphi_{2}\left(x_{f}\right)$ represents the phase distortion applied to DM2.

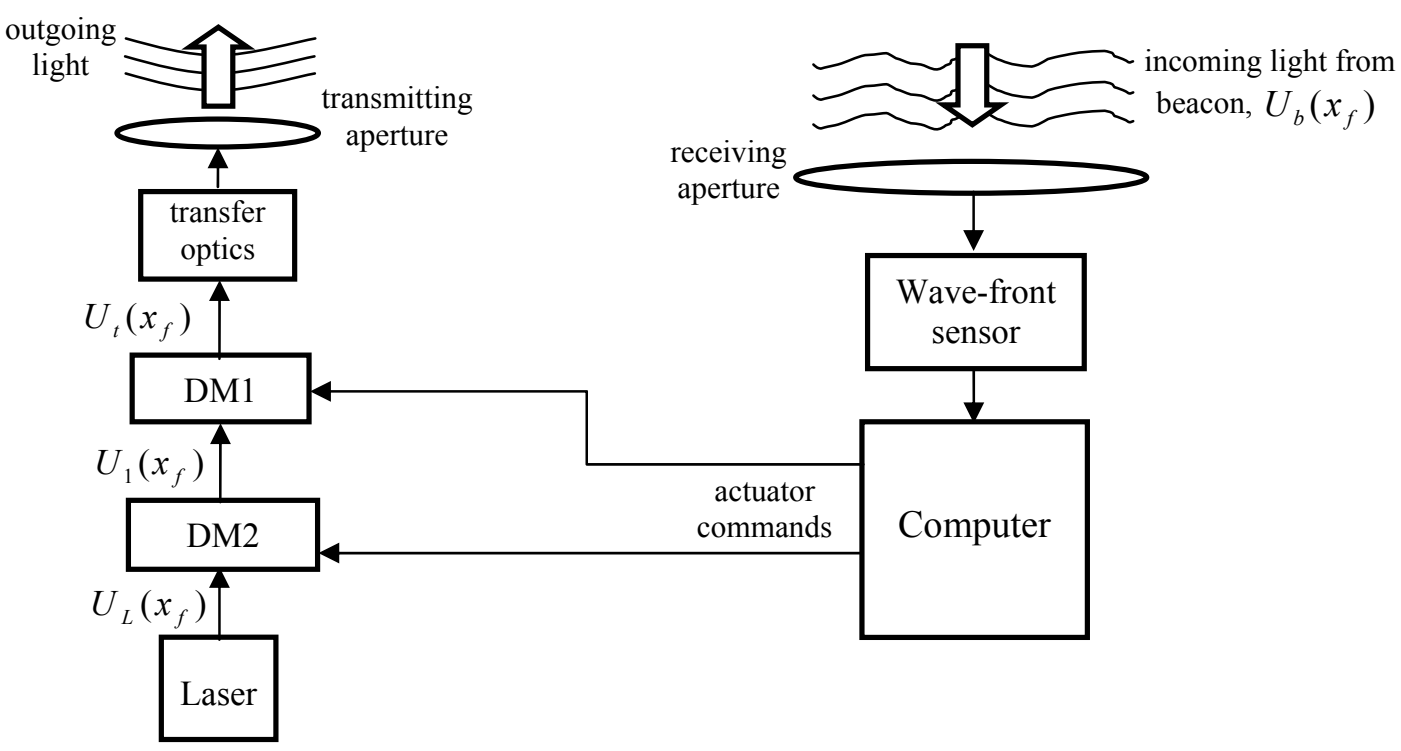

Figure 3. Bistatic configuration for the laser projection system

The purpose of DM1 is to apply a phase distortion to $U_{l}\left(x_{f}\right)$ such that the transmitted wave-front has a phase equal to $\varphi_{b}\left(x_{f}\right)$. (see eq. 3)

The relationship between the phase $\varphi_{1}\left(x_{f}\right)$ applied to DM1 and the amplitude $B\left(x_{f}\right)$ that falls upon DM1 is not linear, so finding the controlled phases $\varphi_{1}\left(x_{f}\right)$ and $\varphi_{2}\left(x_{f}\right)$ requires special numerical approaches such as Phase-Retrieval methods $^{24}$, the Simultaneous Perturbation Stochastic Approximation ${ }^{14}$ or Simulated Annealing algorithms ${ }^{25}$.

The purpose of the AO system in Fig. 3 is to select $\varphi_{1}\left(x_{f}\right)$ and $\varphi_{2}\left(x_{f}\right)$ to correct the aberrated laser field $U_{L}$ and to precompensate for the turbulence-induced distortions as observed in $U_{b}$, maximizing the on-axis intensity of the laser in the far-field. It is assumed that $U_{L}$ and $U_{b}$ are known perfectly and that $\varphi_{1}\left(x_{f}\right)$ and $\varphi_{2}\left(x_{f}\right)$ can take any real value.

There is an additional complication in the calculation of the mirror deformations $\varphi_{1}\left(x_{f}\right)$ and $\varphi_{2}\left(x_{f}\right)$ in that since $B\left(x_{f}\right)$ and $A_{L}\left(x_{f}\right)$ can take small values across the mirror apertures, branch points ${ }^{26}$ are expected to appear. This problem occurs when the field intensity is so weak that the calculation of the field phase becomes highly unreliable, resulting in two-dimensional phase maps that contain abrupt changes that are physically impossible to realize by a continuous plate deformable mirror.

Barchers \& Ellerbroek ${ }^{23}$ propose two methods to solve this problem; the first based on the well-known GerschbergSaxton phase retrieval algorithm ${ }^{27}$ known as the sequential generalized projection algorithm (SGPA) and the second based on the parallel generalized projection algorithm (PGPA) ${ }^{28}$.

Our approach to estimate $\varphi_{1}\left(x_{f}\right)$ and $\varphi_{2}\left(x_{f}\right)$ is based on the procedure described in reference [23] with some modifications to include the laser aberrations, the amplitude shaping of the output beam $U_{t}$ and the avoidance of branch points by using a weighted version of least-squares phase unwrappers ${ }^{29}$.

Using a Fresnel approximation, the free-space propagation of an electromagnetic wave $U_{0}\left(x_{f}\right)$, a distance $z$, is described by the linear, unitary transformation ${ }^{30}$ :

$$
U_{z}\left(x_{f}{ }^{\prime}\right)=T_{z}\left[U_{0}\left(x_{f}\right)\right]=\mathcal{F}^{-1}\left\{\mathcal{F}\left[U_{0}\left(x_{f}\right)\right] \exp \left(j \pi \lambda z k^{2}\right)\right\}
$$


Here $\mathcal{F}$ denotes the two-dimensional Fourier transform operator, $\lambda$ is the wavelength of the light, $x_{f}$ is the variable of integration in the projection plane, and $k$ is the spatial-frequency vector $\left(k=\left[1 /\left(N \Delta x^{2}\right)\right)\right] r$, where $N$ is the grid size and $\Delta x$ is the grid spacing).

Barchers \& Ellerbroek ${ }^{23}$ show that the optimal value of $\varphi_{1}\left(x_{f}\right)$ and $\varphi_{2}\left(x_{f}\right)$ that optimizes the on-axis far-field intensity $I$ of the laser beam is given by:

$$
\begin{aligned}
& \underset{\varphi_{1}\left(x_{f}\right)}{\arg \max } I\left[\varphi_{1}\left(x_{f}\right)\right]=\arg \left(U_{b}^{*}\left(x_{f}\right) T_{z}^{*}\left\{U_{L}\left(x_{f}^{\prime}\right) \exp \left[j \varphi_{2}\left(x_{f}^{\prime}\right)\right]\right\}\right) \\
& \underset{\varphi_{2}\left(x_{f}^{\prime}\right)}{\arg \max } I\left[\varphi_{2}\left(x_{f}^{\prime}\right)\right]=\arg \left(U_{L}^{*}\left(x_{f}\right) T_{z}\left\{U_{b}\left(x_{f}\right) \exp \left[j \varphi_{1}\left(x_{f}\right)\right]\right\}\right)
\end{aligned}
$$

Equations (8) and (9) are iteratively computed to converge to the optimal values of $\varphi_{1}\left(x_{f}\right)$ and $\varphi_{2}\left(x_{f}\right)$.

The iterative process described above is impractical, since it involves the calculation of two phases from fields than can become negligible for some points across the aperture, generating branch points that would corrupt the solutions, also affecting the speed of convergence of the method given by equations (8) and (9).

An alternative to dodge this problem is proposed in ${ }^{23}$, using the PGPA method ${ }^{28}$. The technique reduces the number of branch points substantially, but it does not guarantee a complete elimination.

In our case, we have found that using a weighted least squares approach ( $\mathcal{W L S}$ ) to unwrap the phase can effectively eliminate the branch points while giving accurate solutions. Mathematically:

$$
\mathcal{W L S}[\varphi]=\left(G^{T} W^{2} G\right)^{-1} G^{T} W^{2} \mathcal{P} \mathcal{V}[G \varphi]
$$

where $G$ is the discrete representation of the gradient operator, $\mathcal{P} \mathcal{V}$ is the principal-value operator that outputs a phase or a phase gradient that falls in the range $\pm \pi$ and modulus $2 \pi$, and $W$ is a diagonal array of weights.

Weighted least-squares unwrappers are sometimes used to minimize noise or emphasize certain parts of a field. In applications where the desired output field varies, the pseudo inverse cannot be pre-computed because the weighting matrix is not constant. This makes a weighted least-squares unwrapper difficult to implement in real-time systems. The unwrapping problem in our case is limited to cases where the desired field is known, so the pseudo-inverse via truncatedleast-square techniques can be pre-computed for later use. This is the case for $B\left(x_{f}\right)$ and the valid area of DM2 to be illuminated by the laser beam, i.e. $x_{f}$.

\section{SIMULATION RESULTS}

From equations (8) and (9), we see that it is not possible to define an arbitrary intensity shape for the output field, since the amplitude of $U_{b}$ is constant (incoming wavefront under weak turbulence). To solve this problem we define a new variable $\varphi_{10}\left(x_{f}\right)$, which is the correction for DM1 that ensures a constant zero phase in the output field $U_{t}$. The iteration process is then performed assuming that the incoming wavefront has an arbitrary amplitude field $B\left(x_{f}\right)$ with constant phase across the pupil.

Once the iteration has converged to the desired amplitude shape and phase, the estimated turbulence aberration $\hat{\varphi}_{b}$, from the system WFSs is added to the DM1 correction to compensate for the atmospheric aberrations, i.e.:

$$
\varphi_{1}=\varphi_{10}-\hat{\varphi}_{b}
$$

The algorithm described in the previous sections is evaluated for the optical setup in Fig. 4. The aberrated laser beam $U_{L}$ is optically processed by DM2 $\left(\varphi_{2}\right)$ and DM1 $\left(\varphi_{10}\right)$ to initially generate an output field with the form:

$$
U^{\prime}{ }_{t}\left(x_{f}\right)=B\left(x_{f}\right) \exp \left[j \varphi_{0}\left(x_{f}\right)\right],
$$


where $\varphi_{0}\left(x_{f}\right)$ is a zero-valued matrix.

After convergence, the estimated turbulence $-\hat{\varphi}_{b}$ is added to DM1, so

$$
U_{t}\left(x_{f}\right)=B\left(x_{f}\right) \exp \left[-j \hat{\varphi}_{b}\left(x_{f}\right)\right]
$$

The output is driven to the laser launch telescope (LLT) via the uplink relay optics.

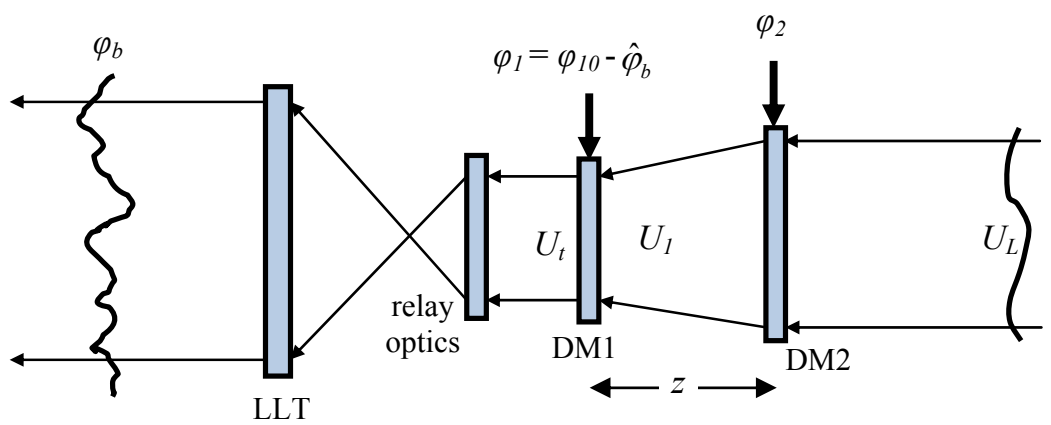

Figure 4. Simulation setup

The aim of the following simulation case is to describe our approach to solve this challenging energy projection problem. It is not in the scope of the paper to carry out a comprehensive analysis looking for an optimal design of the AO system. The latter is left for future work.

We assume an ideal AO system that can sense perfectly the phase $\varphi_{b}$ and the amplitude and phase of the laser field $U_{L}$. Both DMs can also apply perfect correction of the phase.

The incoming turbulence field is generated with a random phase screen, obtained from the Fourier transform of unit variance Gaussian random complex noise weighted by the Kolmogorov turbulence spectrum corresponding to a $r_{0}=10$ $\mathrm{cm}$. The turbulence is assumed to be concentrated at the ground layer. The grid size used is $64 \times 64$ with a resolution of $\Delta \mathrm{x}=0.6 \mathrm{~cm}$ for a launch telescope of $30 \mathrm{~cm}$ in diameter.

Nyquist criterion along the correction and propagation paths ${ }^{23}$ is satisfied in excess with $\Delta \mathrm{x} \ll r_{0}$ and

$$
\left(n_{x}=64\right)>\left(2 \lambda h \sec (\psi) / \Delta x^{2}=29\right),
$$

where $n_{x}$ is the number of grid points on a side, $\lambda$ is the wavelength of propagation $(589 \mathrm{~nm}), h$ is the altitude of the sodium layer (assumed $h=90 \mathrm{~km})$ and $\psi$ is the zenith angle $\left(\psi=0^{\circ}\right)$.

Regarding the two-DM configuration, the diameters are $D_{1}=6 \mathrm{~mm}$ for DM1 and $D_{2}=9 \mathrm{~mm}$ for DM2. Their separation is $z=80 \mathrm{~mm}$.

Figure 5 shows the amplitude and phase for the laser field as approaches to DM2. A "peanut" shape has been defined for the amplitude $A_{L}$ and the phase $\varphi_{L}$ is generated from a combination of different Zernike modes with a peak-to-valley phase difference of $50^{\circ}$.

Figure 6 shows the turbulence at the ground layer cropped to the DM1's pupil.

The challenging shape shown in figure 7 has been selected for the desired amplitude of the outgoing field $B$. and it is given by:

$$
B\left(x_{f}\right)=W_{D M 1}\left(\exp \left[\left(-\left|x_{f}\right| /\left(0.12 D_{1}\right)\right)^{2}\right]-\exp \left[\left(-\left|x_{f}\right| /\left(0.06 D_{1}\right)\right)^{2}\right]\right)
$$

where $W_{D M 1}$ defines the aperture of DM1. 

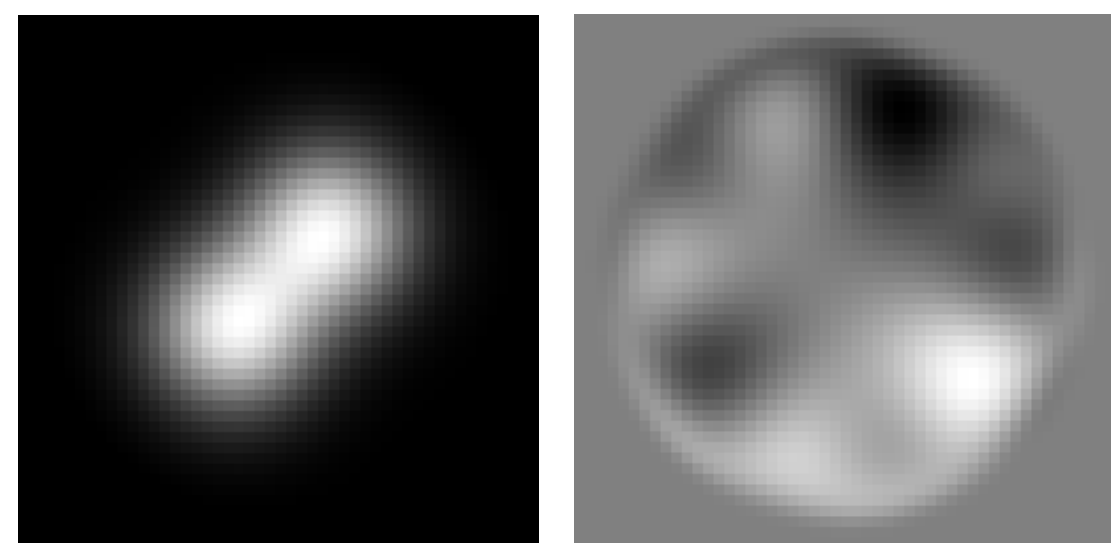

Figure 5. Laser field: amplitude $A_{L}$ (left); phase $\varphi_{L}$ (right)

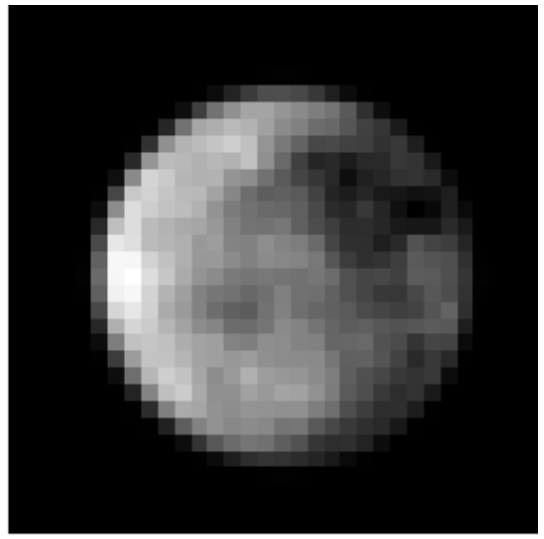

Figure 6. Conjugate turbulence phase $-\hat{\varphi}_{b}$ added to DM1

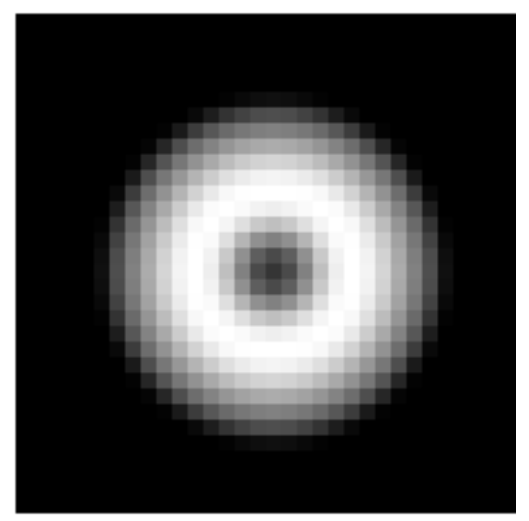

Figure 7. Desired "donut" amplitude shape for output field, $B$

Now the iterative algorithm defined by equation (8) and (9) is applied to the stated problem and as figure 8 shows, a convergence is achieved at around 100 iterations. 


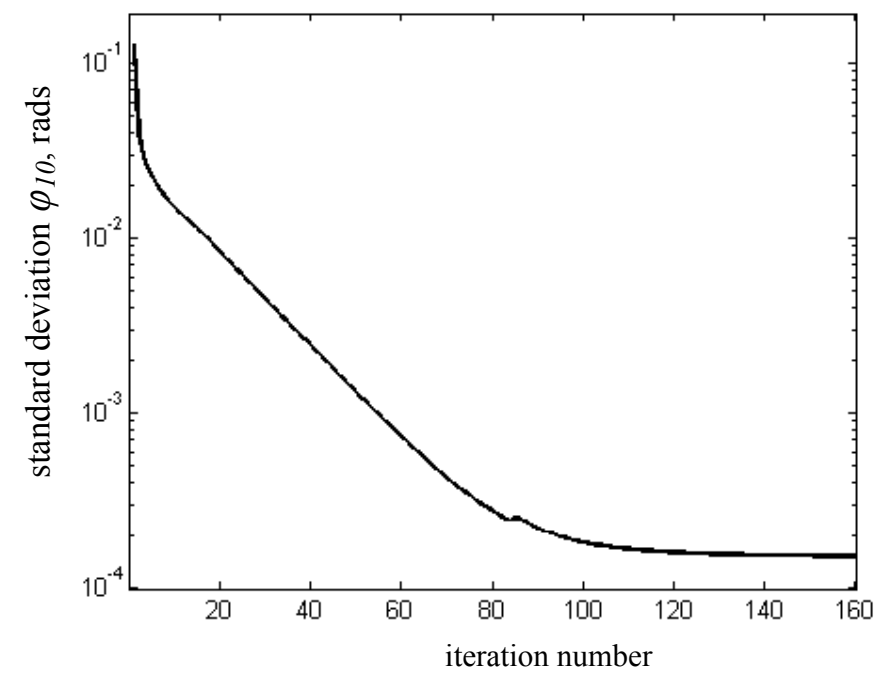

Figure 8. Convergence of the iteration process (standard deviation of $\varphi_{t}$ before adding turbulence conjugate phase $-\hat{\varphi}_{b}$ )

Figure 9 present the results obtained after convergence for the amplitude and phase of $U^{\prime}{ }_{t}$ (before adding turbulence correction phase $-\hat{\varphi}_{b}$ ). A peak-to-peak phase value of approximately 1 minute is obtained for the desired flat phase. This is achieved with the deformations of DM2 and DM1 shown in Fig. 10 where reasonable peak-to-valley corrections of approximately $4 \mu \mathrm{m}$ and $1 \mu \mathrm{m}$ respectively.
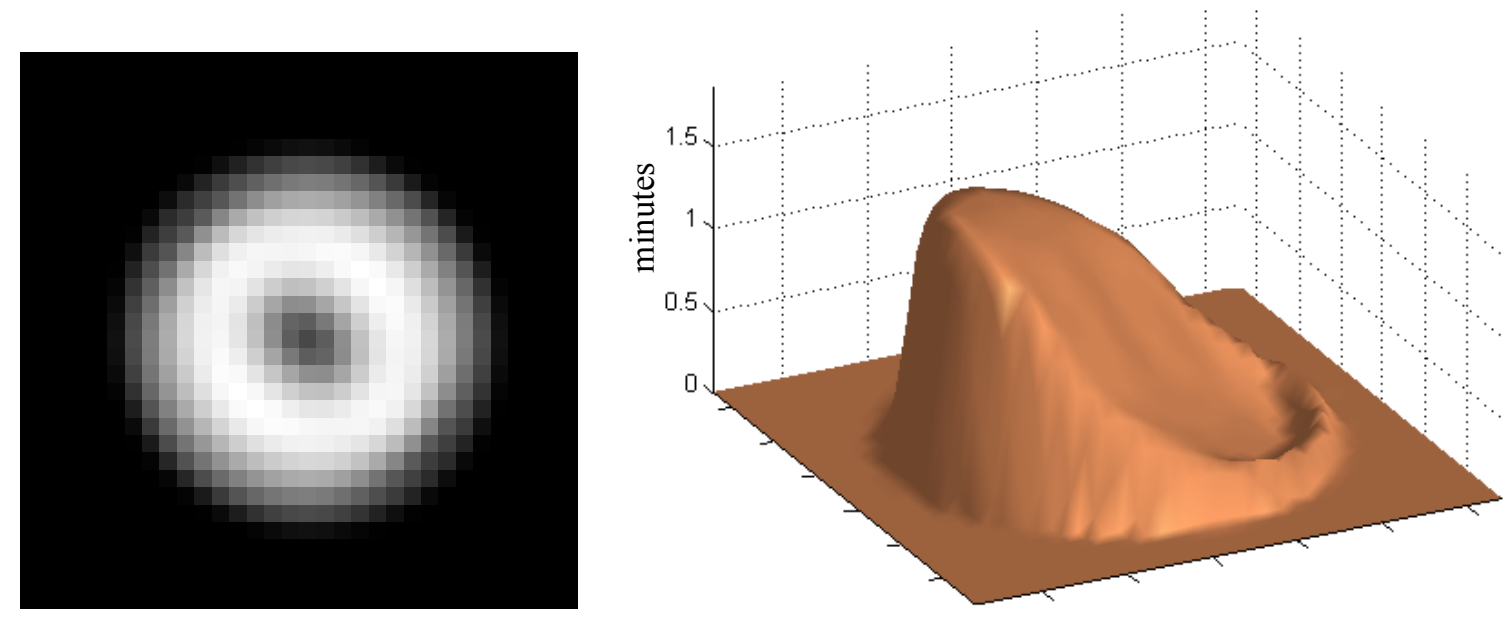

Figure 9. Left: amplitude incident on DM1, $U_{t}^{\prime}$; Right: phase of $U_{t}^{\prime}\left(\right.$ before adding turbulence correction phase - $\left.\hat{\varphi}_{b}\right)$. A peak-topeak phase value of approximately 1 minute is obtained. 

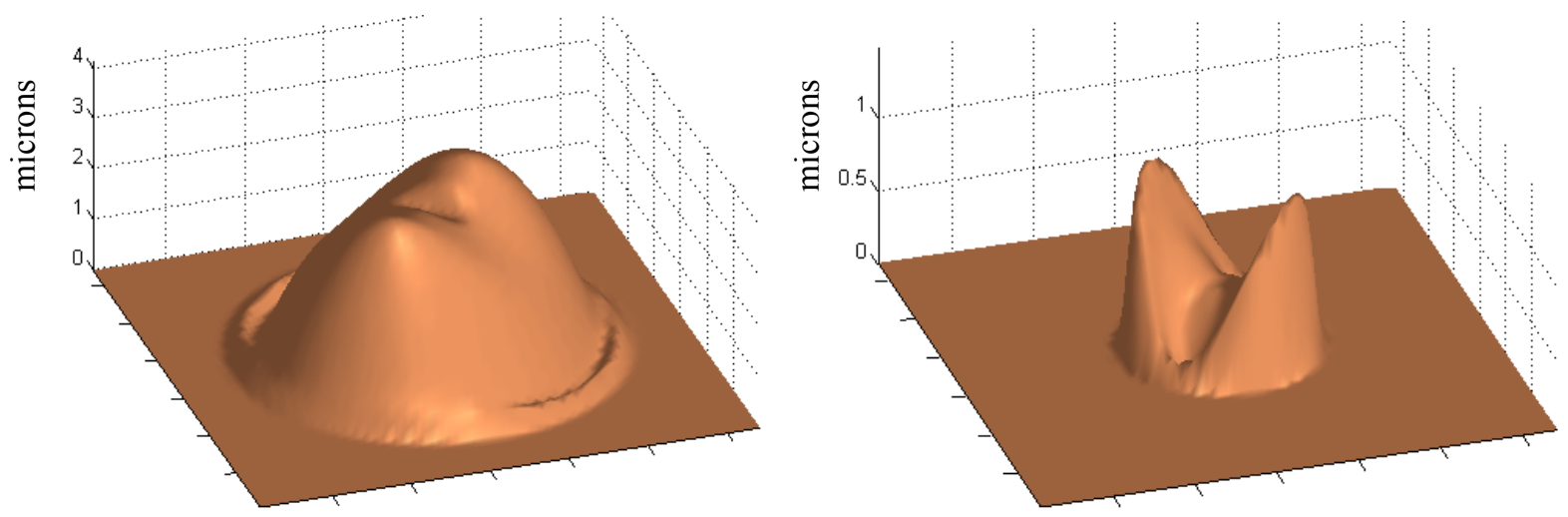

Figure 10. DM surfaces: $\varphi_{2}$ (left) and $\varphi_{10}$ (right)

\section{CONCLUSIONS}

A two deformable mirror system to correct for laser beam aberrations and precompensation of atmospheric turbulence in a laser projection system has been described. The two DMs are required to simultaneously shape the phase and amplitude of the outgoing beam. The use of an iterative process to calculate the DM phases has proved to work with accuracy and robustness. The use of a weighted least square unwrapper has performed extremely well, avoiding branch points and generating smooth surfaces for the DM correcting phases.

Although the setup has been defined with arbitrary parameters, the aim is to implement this technique in the Gemini South MCAO system (GeMS). To achieve this goal, the uncorrected laser field from its source and the atmospheric aberrations must be estimated. The first problem is fairly simple, since being a quasi-static phenomenon, a removable beam-splitter and a wavefront sensor would give the required measurement. However, the problem of estimating the turbulence aberrations is far more complex, since the available WFSs of GeMS do not provide measurement of the turbulence incident on the LLT at low altitudes due to the central obscuration caused by the secondary behind which is the laser launch telescope that requires the precompensation. This limitation will be addressed in future work in order to look for possible solutions to this problem.

\section{ACKNOWLEDGMENTS}

This work has been supported by the Chilean Research Council (CONICYT) through grant Fondecyt 1120626 and Anillo ACT-86.

\section{REFERENCES}

[1] Hardy, J.W., [Adaptive optics for astronomical telescopes], Oxford Series (1998).

[2] Bendek, E., "Status of the 6.5m MMT Telescope laser adaptive optics system", Proc. SPIE 7736, 7736001 (2010).

[3] d'Orgeville, C., Daruich, F., Arriagada, G., Bec, M., Boccas, M., Bombino, S., Carter, C., Cavedoni, C., Collao, F., Collins, P., James, E., Karewicz, S., Lazo, M., Maltes, D., Mouser, R., Perez, G., Rigaut, F., Rojas, R., Sheehan, M., Trancho, G., Vergara, V., Vucina, T., "The Gemini South MCAO laser guide star facility: getting ready for first light", Proc. SPIE 7015, 70152P (2008).

[4] Arsenault, R., Madec, P.Y., Hubin, N., Stroebele, S., Paufique, J., Vernet, E., Hackenberg, W., Pirard, J.F., Jochum, L., Glindemann, A., Jost, A., Conzelmann, R., Kiekebusch, M., Tordo, S., Lizon, J. L., Donaldson, R., Fedrigo, E., Soenke, C., Duchateau, M., Bruton, A., Delabre, B., Downing, M., Reyes, J., Kolb, J., Bechet, C., Lelouarn, M., Bonaccini Calia, D., Quattri, M., Guidolin, I., Buzzoni, B., Dupuy, C., Guzman, R., Comin, M., Silber, A., Quentin, J., La Penna, P., Manescau, A., Jolley, P., Heinz, V., Duhoux, P. and Argomedo, J., "Manufacturing of the ESO adaptive optics Facility", Proc. SPIE 7736, 773620 (2010). 
[5] Chin, J.C.Y., Stalcup, T., Wizinowich, P., Panteleev, S., Neyman, C., Tsubota, K., Summers, D., Stomski, P., Medeiros, D., Nance C., Grace, K., Cooper, A., Wetherell, E., Doyle, S., "Keck I laser guide star AO system integration", Proc. SPIE 7736, 77360V1 (2010).

[6] Rabien, S., Ageorges, N., Barl, L., Davies, R., Deysenroth, M., Gemperlein, H., Genzel, R., Haug, M., Kanneganti, S., Orban de Xivry, G., Thiel, M. and Ziegleder, J., "ARGOS: the laser guide star system for the LBT", Proc. SPIE 7736, 77360E1 (2010).

[7] Wang, L., Otarola, A., Ellerbroek, B., "Impact of laser guide star fratricide on TMT MCAO system", Proc. SPIE 7736, 77360G1 (2010).

[8] Fusco, T., Meimon, S., Michau, V., Petit, C., Robert, C., Conan, J.-M., Clenet, Y., Gratadour, D., Gendron, E., Rousset, G., Cohen, M., Amans, J.-P., Jagourel, P., Schnetler, H., Paufique, J. and Hubin, N., "ATLAS: the EELT laser tomographic adaptive optics system”, Proc. SPIE 7736, 77360D1 (2010).

[9] Shectman, S., Johns, M., “GMT overview”, Proc. SPIE 7733, 77331Y1 (2010).

[10] Amico, P., Campbell, R., Christou, J., "Laser operations at the 8-10m class telescopes Gemini, Keck, and the VLT: lessons learned, old and new challenges", Proc. SPIE 7737, 7737A1 (2010).

[11] Vorontsov M.A., Kolosov, V.V., and Polnau, E., "Target-in-the-loop wavefront sensing and control with a Collett-Wolf beacon: speckle-average phase conjugation", Applied Optics 48, A13 (2009).

[12] Zhao, Z., Lyke, S. D., Roggemann, M. C., "Adaptive Optical Communication through Turbulent Atmospheric Channels", Proc. ICC 5432 (2008).

[13] Roggemann, M.C., Lee, D.J., "Two deformable mirror concept for correcting scintillation effects in laser beam projection through the turbulent atmosphere", Applied Optics 37, 4577 (1998).

[14] Kizito R., Roggemann, M.C., Schulz, T. and Zhang Y., "Image sharpness metric-based deformable mirror control for beam projection systems operating in strong scintillation”, Proc. SPIE 5160, 406 (2004).

[15] Carbon M., "Laser beam shaping in space using adaptive optics", Proc. SPIE, 5087, 83 (2003).

[16] Samarkin, V., Kudryashov, A., "Deformable mirrors for laser beam shaping", Proc. SPIE 7789, 77890B1 (2010).

[17] Henderson, B.G., Mansell, J.D., "Laser beam shaping with membrane deformable mirrors", Proc. SPIE 7093, 7093011 (2008).

[18] Avino S., Potsaid B., and Wen J.T., "Super-Gaussian laser beam shaping using deformable mirrors and intrinsic beam quality metrics", Proc. SPIE 7266, 72660P1 (2008).

[19] Love G.D., "Wave-front correction and production of Zernike modes with a liquid-crystal spatial light modulator", Applied Optics 36, 1517 (1997).

[20] Baker, K. L., Stappaerts, E. A., Gavel, D., Wilks, S. C., Tucker, J., Silva, D. A., Olsen, J., Olivier, S. S., Young, P. E., Kartz, M. W., Flath, L. M., Kruelevitch, P., Crawford, J., Azucena, O., "High-speed horizontal-path atmospheric turbulence correction with a large-actuator-number microelectromechanical system spatial light modulator in an interferometric phase-conjugation engine", Optics Letters 29, 1781 (2004).

[21] Roggemann M.C., Welsh B., "Imaging through turbulence”, CRC Press, Boca Raton (1996).

[22] Schmitt M.J., "Mitigation of thermal blooming and diffraction effects with high-power laser beams", J. Opt. Soc. Am. B 20, 719-724 (2003).

[23] Barchers J. D., Ellerbroek, B. L., "Improved compensation of turbulence-induced amplitude and phase distortions by means of multiple near-field phase adjustments", J. Opt. Soc. Am. A, 18, 399 (2001)

[24] Eismann M.T., Tai A.M., and Cederquist J. N., "Iterative design of a holographic beamformer", Applied Optics 28, 2641 (1989).

[25] El-Agmy, R., Butle, H., Greenaway, A. H., and Reid, D. T., "Adaptive beam profile control using a simulated annealing algorithm", Optics Express 13, 6085 (2005).

[26] Fried, D., "Branch point problem in adaptive optics", J. Opt. Soc. Am. A 15, 2759 (1998).

[27] Gerchberg, R. W. and Saxton, W. O., "A practical algorithm for the determination of phase from image and diffraction plane images", Optik 35, 225 (1972).

[28] Kotzer, T., Rosen J., and Shamir J., "Application of serial and parallel projection methods to correlation filter design”, Applied Optics 34, 3883 (1995).

[29] Venema, T. M., and Schmidt J. D., "Optical phase unwrapping in the presence of branch points", Optics Express 16, 6985 (2008).

[30] Goodman J. W., "Fourier Optics", McGraw-Hill, New York, (1968). 